\title{
Phase transitions in pseudospin- electron model with direct interaction between pseudospins
}

\author{
I.V.Stasyuk, Yu.Havrylyuk \\ Institute for Condensed Matter Physics \\ of the National Academy of Sciences of Ukraine, \\ 1 Svientsitskii Str., 79011 Lviv, Ukraine
}

Received October 12, 1998

The analysis of thermodynamic properties of the pseudospin-electron model in the case of zero electron transfer with the inclusion of the direct pseudospin-pseudospin interaction of ferroelectric type is performed. The equilibrium conditions in the regimes $\mu=$ const and $n=$ const are investigated in the mean field approximation. It is shown that the interaction with electrons leads at the fixed $\mu$ value to the possibility of the first order phase transition at the change of temperature with a jump-like behaviour of $\left\langle S^{z}\right\rangle$. In the regime $n=$ const there takes place an instability with respect to phase separation in the wide range of asymmetry parameter $h$ values.

Key words: pseudospin-electron model, phase transitions, phase separation

PACS: $74.65 .+n, 71.45 . \mathrm{Gm}$

\section{Introduction}

Pseudospin-electron model (so called Muller model [1]) is the one of theoretical models which were proposed in connection with the investigation of characteristic features of electron spectrum and lattice dynamics in high temperature superconductors. In this model strong Hubbard-type electron correlations are taken into account. Pseudospin formalism is used to describe the locally anharmonic lattice vibrations.

Hamiltonian of the model is of the following form $[2,3]$

$$
\begin{gathered}
H=\sum_{i} H_{i}+\sum_{i j \sigma} t_{i j} a_{i \sigma}^{+} a_{i \sigma}-\frac{1}{2} \sum_{i j} J_{i j} S_{i}^{z} S_{j}^{z}, \\
H_{i}=U n_{i \uparrow} n_{i \downarrow}-\mu \sum_{\sigma} n_{i \sigma}+g \sum_{\sigma} n_{i \sigma} S_{i}^{z}+\Omega S_{i}^{x}-h S_{i}^{z},
\end{gathered}
$$


where in the single site part in addition to Hubbard correlation $U$ there are terms that describe tunnelling splitting and asymmetry of local anharmonic potential (longitudinal field $h$ ). Hamiltonian (1) also contains terms, which describe electron transfer $t_{i j}$ and direct interaction between pseudospins $J_{i j}$. The energy is accounted from the level of chemical potential .

Most attention while investigating this model has been paid to examining the electron states, an effective electron-electron interaction, to studying the dielectric instabilities or ferroelectric type anomalies as well as the tendency to the spatially modulated charge and pseudospin orderings $[4,5]$.

This work is devoted to the study of thermodynamics of the pseudospin-electron model in the case of zero electron transfer $\left(t_{i j}=0\right)$ and zero frequency of tunnelling splitting $(\Omega=0)$. The direct interaction between pseudospins is supposed to be a long-ranged $\left(J_{i j} \sim J / N\right)$ that makes it possible to use the mean field approximation (MFA).

In this approximation, the Hamiltonian of the model is of the form

$$
\begin{aligned}
H & =\sum_{i} \tilde{H}_{i}+\frac{N}{2} J \eta^{2} \\
\tilde{H}_{i} & =-\mu \sum_{\sigma} n_{i \sigma}+U n_{i \uparrow} n_{i \downarrow}+g \sum_{\sigma} n_{i \sigma} S_{i}^{z}-(h+J \eta) S_{i}^{z} .
\end{aligned}
$$

The interaction $J_{i j}$ is taken because the ferroelectric type interaction $(J(\vec{q})$ has got a maximum value at $\vec{q}=0 ; J(0) \equiv J>0)$; the order parameter $\eta=\left\langle S_{i}^{z}\right\rangle$ does not depend on the unit cell index.

We investigate the possible states and phases of the system as well as the transitions between them at the change of temperature and model parameters values.

\section{Mean field approximation; $\Omega=0$}

Thermodynamic potential of the model, calculated per one lattice site is equal in MFA to

$$
\begin{aligned}
& \frac{\Omega}{N}=-\theta \ln Z_{i}+\frac{1}{2} J \eta^{2} \\
& Z_{i}=2\left[\cosh \beta H+\mathrm{e}^{-\beta(U-2 \mu)} \cosh \beta(H-g)+2 \mathrm{e}^{\beta \mu} \cosh \beta\left(H-\frac{g}{2}\right)\right] .
\end{aligned}
$$

To investigate the equilibrium conditions we separate two regimes: $\mu=$ const and $n=$ const. The equilibrium for these regimes is defined by the minimum of thermodynamical potential $\left((\partial \Omega / \partial \eta)_{T, \mu, h}=0\right)$ or the minimum of free energy $F=$ $\Omega+\mu N\left((\partial F / \partial \eta)_{T, \mu, h}=0\right)$ respectively.

The equation for the order parameter is obtained from these conditions in the form

$$
\eta=\frac{1}{2} \frac{\sinh \beta H+\mathrm{e}^{-\beta(U-2 \mu)} \sinh \beta(H-g)+2 \mathrm{e}^{\beta \mu} \sinh \beta\left(H-\frac{g}{2}\right)}{\cosh \beta H+\mathrm{e}^{-\beta(U-2 \mu)} \cosh \beta(H-g)+2 \mathrm{e}^{\beta \mu} \cosh \beta\left(H-\frac{g}{2}\right)} .
$$


The average number of electrons $n=\left\langle\frac{1}{N} \sum_{i \sigma} n_{i \sigma}\right\rangle$ is determined as follows:

$$
n=-\frac{1}{N}\left(\frac{\partial \Omega}{\partial \mu}\right)_{T, h}=\frac{4}{Z_{i}}\left[\mathrm{e}^{-\beta(U-2 \mu)} \cosh \beta(H-g)+\mathrm{e}^{\beta \mu} \cosh \beta\left(H-\frac{g}{2}\right)\right] .
$$

The equation (5) and expression (6) form a set of equations for the parameter $\eta$ and for the chemical potential.

After eliminating the chemical potential, the equation for the order parameter transforms to the following form:

$$
\begin{aligned}
& \eta=\frac{1}{2}\left(\frac{n}{2}-\frac{\delta}{2}\right) \operatorname{th} \beta(H-g)+\frac{1}{2} \delta \operatorname{th} \beta\left(H-\frac{g}{2}\right) \\
& +\frac{1}{2}\left(\frac{2-n}{2}-\frac{\delta}{2}\right) \operatorname{th} \beta H
\end{aligned}
$$

where

$$
\delta=\frac{(2-n) a y}{b+a y}=\frac{\sqrt{(1-n)^{2}+n(2-n) \mathrm{e}^{-\beta U} \frac{b}{a^{2}}}-1}{\mathrm{e}^{-\beta U} \frac{b}{a^{2}}-1},
$$

and the $b / a^{2}$ ratio, is given by the expression

$$
\frac{b}{a^{2}}=\frac{\cosh \beta g+\cosh \beta(2 H-g)}{1+\cosh \beta(2 H-g)} .
$$

The equations (5) and (7) determine the values of $\eta$ parameter, which correspond to the extremuma of thermodynamical potential $\Omega$ and free energy $F$ respectively. From the set of all possible roots of it, it is necessary to take into consideration only those which provide the minimum values of $\Omega$ or $F$.

\section{The equation of state $\eta=\eta(h)$ and phase diagrams at $T=0$ (regime $n=$ const)}

The equation for the parameter $\eta$, obtained in the regime of fixed number of electrons, can be solved analytically in the limit $T=0$.

Typical example of $\eta(h)$ dependence is shown in figure 1, which corresponds to the $n$ value in the interval $0 \leqslant n \leqslant 1$. At some regions of values of $h$, where $\eta(h)$ function possesses S-like behaviour and has got three or more values, the first order phase transitions with the jumps of parameter take place at the change of $h$. Phases $\eta=-\frac{1}{2}$ (phase 1), $\eta=\frac{1}{2}-n$ (phase 2), $\eta=\frac{1}{2}-\frac{n}{2}$ (phase 3$), \eta=\frac{1}{2}$ (phase 4 ) occur between phase transition points and outside them. At the change of parameter values the regions, where metastable phases occur, can overlap. The disappearing of some phase transitions takes place and therefore some intermediate phases are not realizable. In case $1 \leqslant n \leqslant 2$ the dependence of $\eta(h)$ is generally similar. The phase 3 and phase 2 ' at $\eta=3 / 2-n$, which now appears instead of phase 2 , may play the role of intermediate phases. 


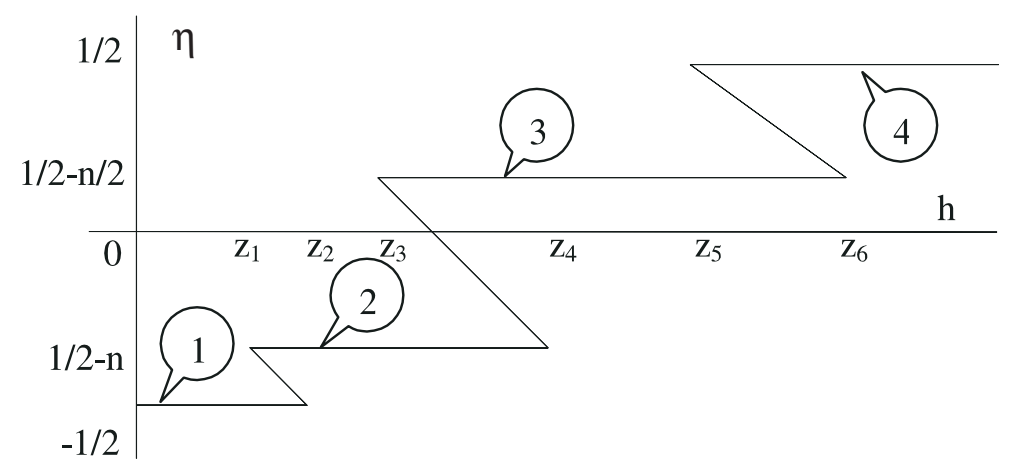

Figure 1. The dependence of $\eta$ parameter on $h$ field at $T=0$ (in the case $g>\frac{J}{2}$; $\left.\frac{J}{2}-\frac{n J}{4}<U<g-\frac{n J}{4} ; 0<n<1\right)$

The analysis shows that the states described by $\overline{1}\left(z_{1} \leqslant h \leqslant z_{2}\right), \overline{2}\left(z_{3} \leqslant h \leqslant z_{4}\right)$, $\overline{3}\left(z_{5} \leqslant h \leqslant z_{6}\right)$-lines are unstable while the states $1 \ldots 4$ correspond to the minimum values of the free energy. The intersections of $F_{1}, F_{2}, F_{3}, F_{4}$ lines determine the points of the first order phase transition between relevant phases. Some of these phase transitions will not be realizable, if the relevant points of crossing lie above some other $F_{k}$-line.

The values of field $h=h_{i k}$, at which phase transitions $i \leftrightarrow k$ occur, are the following:

$$
\begin{array}{ll}
h_{12}=\frac{n J}{2} ; & h_{23}=U-\frac{J}{2}+\frac{3 n}{4} J ; \\
h_{34}=2 g-U-\frac{J}{2}+\frac{J n}{4} ; & h_{13}=\frac{n J}{4}+\frac{n}{2-n} U ; \\
h_{24}=g-\frac{1-n}{2} J ; & h_{14}=n g
\end{array}
$$

at $0 \leqslant n \leqslant 1$, and

$$
\begin{array}{ll}
h_{12}=g-\frac{1-n}{2} J ; & h_{23}=2 g-U-J+\frac{3 n}{4} J ; \\
h_{13}=U+\frac{J_{n}}{4} ; & h_{12}=2 g-\frac{J}{2}+\frac{J n}{4}-\frac{2-n}{n} U ; \\
h_{2^{\prime} 4}=2 g-J+\frac{J n}{2} J ; & h_{14}=n g
\end{array}
$$

at $1 \leqslant n \leqslant 2$.

Some examples of phase diagrams $(n, h)$ where the areas of occurrence of the phases $1 \ldots 4$ at $T=0$ are shown, are presented in figure $2^{1}$. Their emerging depends on the values of $U$ and $g$ constants. In addition, they show the possibility of transforming the phases at the change of electron concentration $\mathrm{n}$. To study this problem more attentively, we investigated the dependence $\mu(n)$ described by the equation (6).

It is known that the dependence of particle concentration on chemical potential is one of the factors that determines thermodynamical equilibrium of the system. The state with homogenous distribution of particles is unstable at $\left(\frac{\partial n}{\partial \mu}\right)_{T}<0$. Hence, the phase separation into the regions with different concentrations takes place.

Let's take, for instance, the case $U>g, 0<h<g$; the corresponding phase diagram in plane $(n, h)$ is shown on figure $2 \mathrm{a}$. One can see that in phases 1 and 4

\footnotetext{
${ }^{1}$ The $(n, h)$ and $(U, h)$ phase diagrams for all possible cases are given in $[6]$
} 
a)

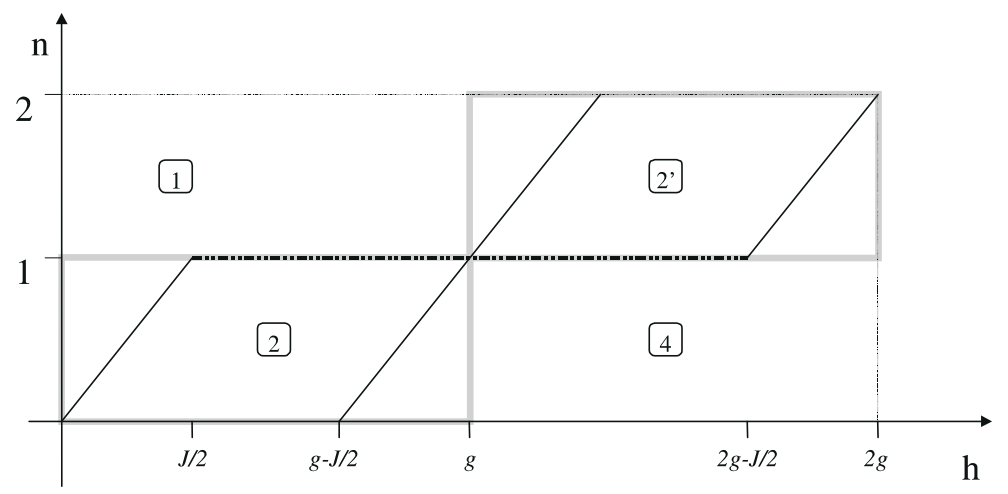

b)

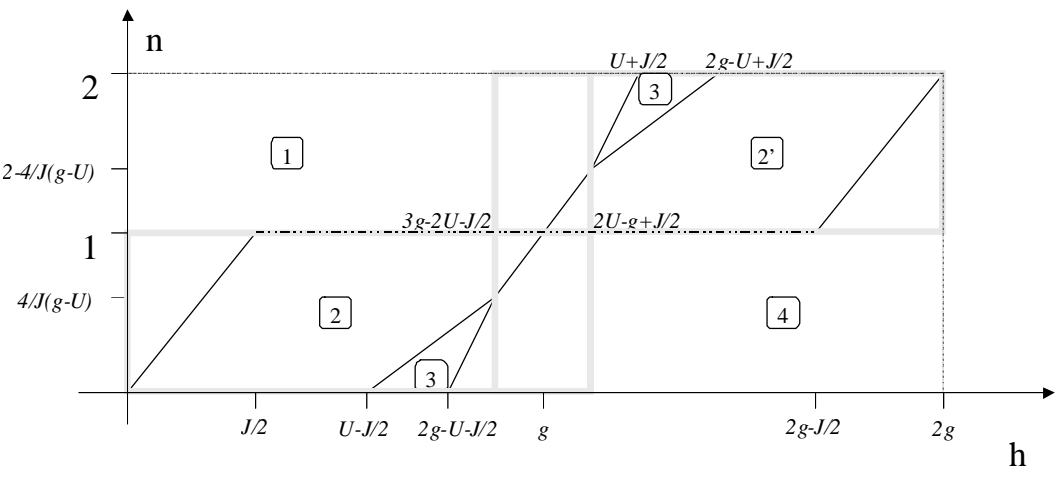

Figure 2. $(n, h)$ - phase diagrams at $T=0$; a) $U>g$; b) $g-\frac{J}{4}<U<g$;

the chemical potential increases (or remains constant) with the increase of $n$, while within the phase 2 there is a possibility of descending dependence of $\mu$ on $n$. That

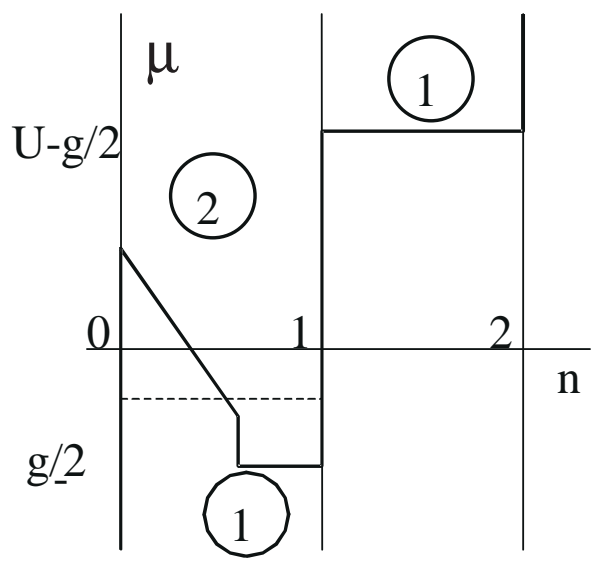

Figure 3. The dependence of chemical potential on the electron concentration $n(T=0) . U>g$; $g>J / 2 ; 0<h<J / 2$. means the instability of homogenous state of the system. In the case illustrated on figure 3 the phase 2 splits at $T=0$ into phase $4(n=$ $\left.0, \eta=\frac{1}{2}\right)$ and into phase $1\left(n=1, \eta-\frac{1}{2}\right)$ with weight coefficients $1-n$ and $n$ respectively.

Phase separated states are bordered by binodal lines. For example, at $T=0$ and $U>g$ they are:

$$
\begin{array}{ll}
n=0, & (0<h<g) \\
n=1, & (0<h<2 g) \\
n=2, & (g<h<2 g) .
\end{array}
$$

Such boundaries surround the regions of occurring of the intermediate phases 2, 2', and 3. The mentioned phases might be stable only if there were some factors that maintain the space homogeneity of electron concentration. 


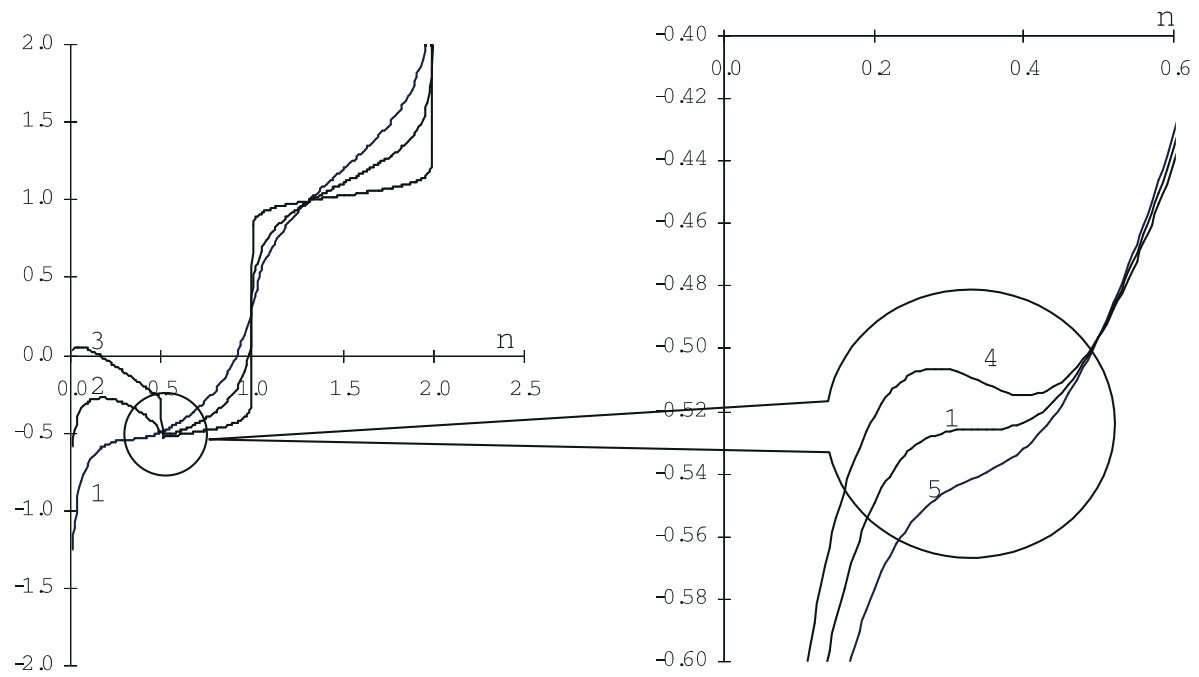

Figure 4. The $n$ dependence of chemical potential $\mu$. The parameter values: $g / J=1.0 ; U / J=1 ; h / J=0.25 ;[1-T / J=0.25,2-T / J=0.14,3-$ $T / J=0.03,4-T / J=0.24,5-T / J=0.26] . \mu$ is given in dimensionless units: $\mu \rightarrow \mu / J$.

\section{Phase transition at $\mu=$ const}

The dependence of the order parameter on field $h$ and temperature $\theta$, at the constant value of chemical potential, is determined according to equation (5).

Here we consider the case of non-zero temperature. Among all possible solutions $\eta=f(\theta, h, \mu)$ of equation (5), let us separate the zero ones. In $(\theta, h)$-plane they define the curves which are described by an equation

$$
\sinh \left(\frac{\beta h}{2}\right)+\mathrm{e}^{-\beta(U-2 \mu)} \sinh \beta\left(\frac{h}{2}-g\right)+2 \mathrm{e}^{\beta \mu} \sinh \beta\left(\frac{h}{2}-\frac{g}{2}\right)=0 .
$$

From this equation one can get

$$
h=\theta \ln \frac{\xi_{1}}{\xi_{2}}
$$

where

$$
\begin{aligned}
& \xi_{1}=1+\mathrm{e}^{-\beta(U-g-2 \mu)}+2 \mathrm{e}^{\beta\left(\frac{g}{2}+\mu\right)}, \\
& \xi_{2}=\left.\xi_{1}\right|_{g \rightarrow-g} .
\end{aligned}
$$

After substituting this expression for field $h$ into equation (10) we get an equation $\eta=\frac{1}{2} \operatorname{th} \frac{\beta J \eta}{2}$, that defines the order parameter along the line $h=\theta \ln \frac{\xi_{1}}{\xi_{2}}$ and has got the standard form of molecular field equation. In addition to zero solution, non-zero ones occur at $\theta<\theta_{c}=\frac{J}{4}$. One can make a conclusion that at the temperatures $\theta<\theta_{c}$ the relation (14) has the meaning of equation that describes the line of phase equilibrium (first order phase transition curve), and temperature $\theta_{c}$ corresponds to the critical point. Critical value of $h_{\mathrm{c}}$ is given by the expression

$$
h_{c}=\frac{J}{4} \ln \frac{1+\mathrm{e}^{-\frac{4}{J}(U-g-2 \mu)}+2 \mathrm{e}^{\frac{4}{J}\left(\frac{g}{2}+\mu\right)}}{1+\mathrm{e}^{-\frac{4}{J}(U+g-2 \mu)}+2 \mathrm{e}^{-\frac{4}{J}\left(\frac{g}{2}-\mu\right)}} .
$$


The feature of this phase transition is that the curve of phase equilibrium generally is not parallel to the temperature axis. The occurrence of a bent in the cooccurrence curve testifies to the possibility of the first order phase transition at the change of temperature with a jump of the order parameter $\eta$ if the value of field $h$ is within the interval placed between $2 H^{*}$ and $h_{\mathrm{c}}$ values.

At the temperatures below the critical value crossing the phase coexistence curve leads to the jump of electron concentration between values that correspond to phases, which are involved in phase separation (this corresponds to the break point on the dependence $\Omega(\mu)$ ). At the same time these values are points of binodal lines, which are determined according to the Maxwell rule from the plot of function $\mu(n)$. An illustrative sample of this is presented in figure 4 . The plots presented are obtained using numerical calculations based on formulas (5) and (6).

\section{Conclusions}

The investigation performed shows that pseudospin-electron model with a longrange interaction possesses some features that make it different from the ordinary Ising model. They are as follows:

- The possibility of the first order phase transition occurring at the change of temperature and at fixed value of the field (regime $\mu=$ const)

- Instability with respect to phase separation in the wide range of parameter $h$ (regime $n=$ const) with the emerging of the regions with different electron concentration and different orientation of pseudospins.

The results obtained give a more substantiated interpretation of the behaviour of the pair correlation function and dielectric susceptibility of the model in the case $t \neq$ $0, J=0$ investigated in GRPA [5,7]. It is possible to conclude that the divergence of susceptibility $\left.\chi_{s s}\right|_{\mu=\text { const }}$ and other related quantities at certain values of temperature and $h \sim g($ at $U=\infty)$ corresponds to the point of the high-temperature phase instability. The temperature of this instability is situated below the temperature of the first order phase transition that leads to the jump of $\left\langle S^{z}\right\rangle$.

When the model is used to describe the thermodynamics of oxygen subsystem in $\mathrm{YBa}_{2} \mathrm{Cu}_{3} \mathrm{O}_{7}$-crystals, the results of this work might be used as a basis for describing the bistability phenomena in apex oxygen sublattices as well as for studying the experimentally observed spatial nonuniformities and phase-separated states in monocrystalline specimens $[8,9]$.

This work was partially supported by the Ministry of Ukraine for Science and Technology (project No. 2.4/171). The authors express their gratitude to O.Velychko for the assistance in the preparing the manuscript.

\section{References}

1. Muller K.A. Phase transitions. // 1988 (Special issue). 
2. Frick M., von der Linden W., Morgenstern I., Raedt H. Local anharmonic vibrations, strong correlations and superconductivity. A quantum simulation study. // Z. Phys. B. Condensed Matter, 1990, vol. 81, No. 2, p. 327-335.

3. Stasyuk I.V., Shvaika A.M., Shachinger E. On the electron spectrum of the Hubbard model including interactions with local anharmonic vibrations. // Physica C, 1993, vol. 213, No. 1, p. 57-70.

4. Stasyuk I.V., Shvaika A.M. Electron spectrum and dielectric susceptibility of the Hubbard model with local lattice anharmonicity. // Acta Physica Polonica A, 1994, vol. 85, No. 2, p. 363-366.

5. Stasyuk I.V., Shvaika A.M., Danyliv O.D. Dielectric instability and charge ordering in the local anharmonic model of high- $T_{\mathrm{c}}$ superconductors. // Molecular Physics Reports, 1995, vol. 9, p. 61-75.

6. Stasyuk I.V., Havrylyuk Yu. Phase transitions in pseudospin-electron model with direct interaction between pseudospins. // Preprint of the Institute for Condensed Matter Physics, ICMP-98-18E, Lviv, 1998, 20 p.

7. Stasyuk I.V., Shvaika A.M. A model with local anharmonicity in theory of HTSC systems: correlation functions and "transverse" dielectric susceptibility. // Condensed Matter Physics, 1994, vol. 3, p. 134-175.

8. Browning V.M. et al. Structural inhomogeneities observed in $\mathrm{YBa}_{2} \mathrm{Cu}_{3} \mathrm{O}_{7-\delta}$ crystals with optimal transport properties. // Phys. Rev. B, 1997, vol. 56, p. 2860.

9. Iliev M.N., Hadjiev V.G., Ivanov V.G. Raman spectroscopy of local structure and reordering processes in $\mathrm{YBa}_{2} \mathrm{Cu}_{3} \mathrm{O}_{7-\delta}$-type compounds. // Journ. of Raman spectroscopy, 1996, vol. 27, p. 333-342.

\title{
Фазові переходи у псевдоспін-електронній моделі з прямою взаємодією між псевдоспінами
}

\author{
І.В.Стасюк, Ю.Гаврилюк \\ Інститут фізики конденсованих систем НАН України, \\ 79011 Львів, вул. Свєнціцького, 1 \\ Отримано 12 жовтня 1998 р.
}

Проведено аналіз термодинамічних властивостей псевдоспін-електронної моделі у випадку відсутності електронного переносу з включенням прямої взаємодії між псевдоспінами сегнетоелектричного типу. У наближенні середнього поля досліджуються умови рівноваги в режимах $\mu=$ const та $n=$ const. Показано, що взаємодія з електронами при фіксованому значенні $\mu$ приводить до можливості фазового переходу першого роду при зміні температури зі стрибкоподібною зміною $\left\langle S^{z}\right\rangle$. У режимі $n=$ const має місце нестабільність щодо розділення фаз у широкому інтервалі значень параметра асиметрії $h$.

Ключові слова: псевдоспін-електронна модель, фазові переходи, розділення фаз

PACS: $74.65 .+n, 71.45 . \mathrm{Gm}$ 